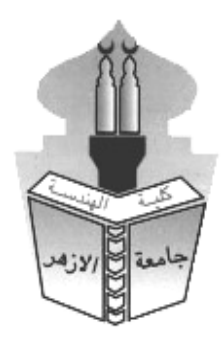

\title{
ARCHITECTURAL FORMING BETWEEN FORM MAKING AND FORM FINDING TOWARDS FORM FOLLOWS PARAMETERS
}

\begin{abstract}
Generating architectural forms are based on variant techniques or methodologies of forming. Though, all of these techniques are derived from either a form making process or a form finding process. Intuition and unpredictability are the essence of the form-making process. Form making is a process of embodying ideas directly into forms. In a forming process characterized by coincidence and no logic or using imitation and metaphor. Mathematical rules had always played a significant role in the process of creation of form in art and architecture through history. Using such a rule based or constrained form seeking methodology is called form-finding process.
\end{abstract}

The reciprocity between mathematics and IT elaborates new mathematical tools with different activity, rather than creating variants of functional solutions, drafting, modeling and presenting, but also generating forms. This approach is known by form-generation process. It had been developed from using only rules and algorithms in the architectural forming process in a one-way process, to using a full range of parametric equations that can be recorded to return to any parameter in any of the design stages to change it and change the whole design solution as well in a glance. Nowadays, forms tend to follow parameters and function. Distinguishing the difference between those form-seeking methodologies through an analytical comparison disclosing the applications and examples of both methodologies is the aim of this paper.

\section{KEYWORDS: Form Making, Form Finding, Form Generation, Parametric Modeling.}




\section{Form making}

Form Making is a process of creation using intuition, that is the ability to foresee without trying to understand, and imagination, that is the ability to create certain images in our mind. Form precedes analysis and design constrains.

The key relationship between designer and product is a direct one (even if mediated via a thirdparty or medium wither conventional or computational). Product Cases or methodologies in

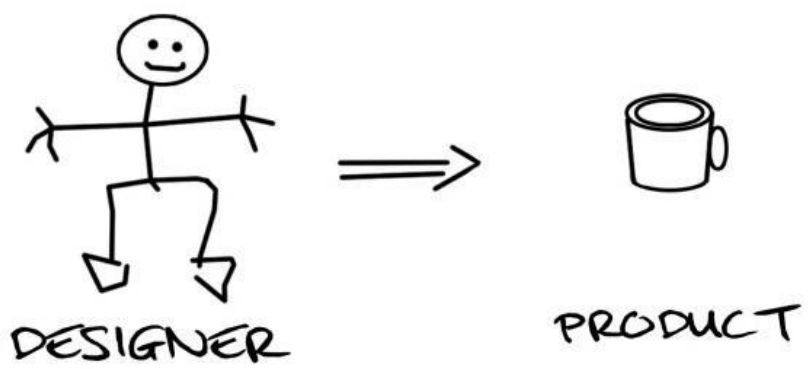

which mental construct arises first are labeled form-making, where designers "have idea" then sketch it (Fig. 1). Extreme form-making is not architecture but sculpture, perhaps form without

Function

Fig.1 form-making process

\subsection{Analogue Form-making}

Depending on the conventional sources of inspiration and the essences and theories of creativity all over the decades that were based on: Intuition, unpredictability, metaphorization and no logic. Theories of incubation elaborated by (Wallas), Genploration (Finke, Ward and Smith), Redundant generation (Lem), and Synectics (Gordon). All these theories emphasize the role of Intuition, Coincidence, unpredictability and metaphors in the form making process.

\subsubsection{Using Coincidence}

An example showing Form-Making of an abstract dynamic form by coincidence (Fig.2). For this purpose, pieces of folded papers and cardboards were used. 2D composition was created on the scanner surface and scanned. The images were achieved by coincidence, however not without the intervention of the authors, who were responsible for choosing the amount and the quality of the elements used.The previous example composition was transformed into 2D linear drawing. Saved picture was opened in the AutoCAD, where it was converted into 3D wired form. The next step was visualization in the 3D Studio. In the process of searching of new idea of architectural form was used simple graphic software, which enables to elaborate the linear pictures similar to the handmade sketch. In all cases find results were by coincidence and unpredictable.

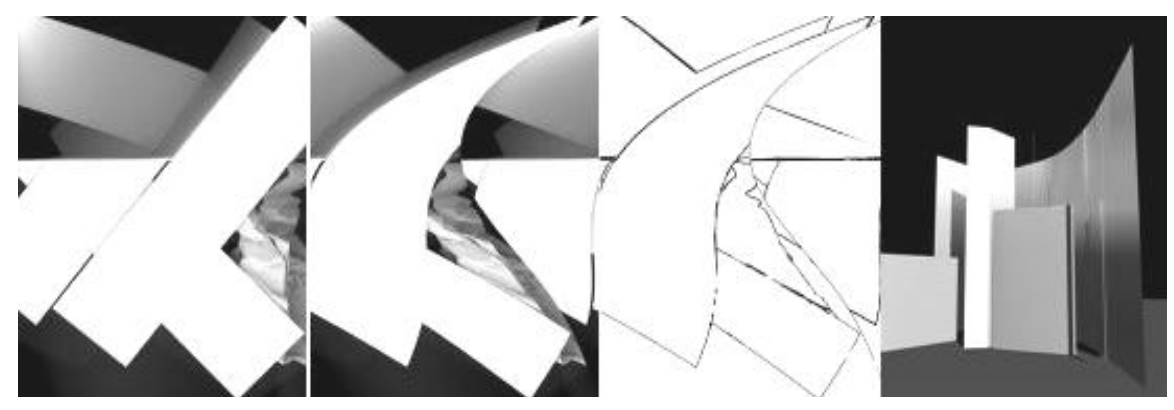




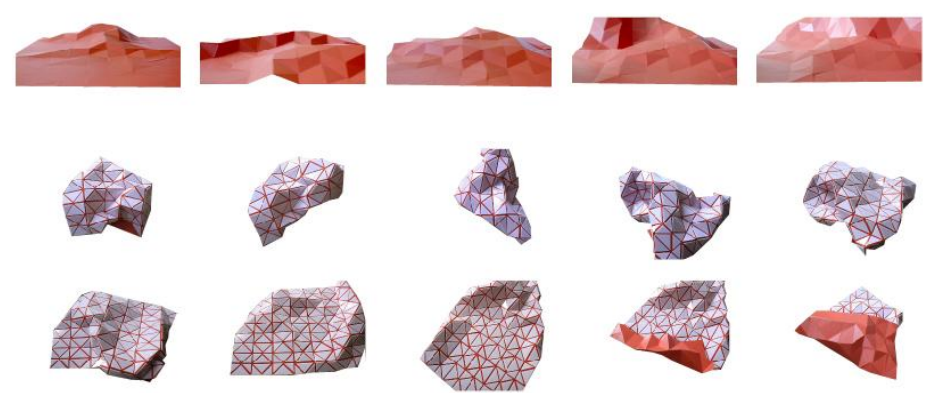

Fig. 2: Process of form making using folded paper \& cardboard

\subsubsection{Using analogy and imitation of nature}

Many architects and designers have explored the interface between architecture and nature (Fig. 3). In his Patterns of Nature, Peter Stevens revealed the geometric patterns present in nature. He juxtaposes the branching of trees with branching arteries and rivers; images of crystal grains and soap bubbles. To the casual observer, nature appears limitless in its ability to trigger and empower the Form-making process.
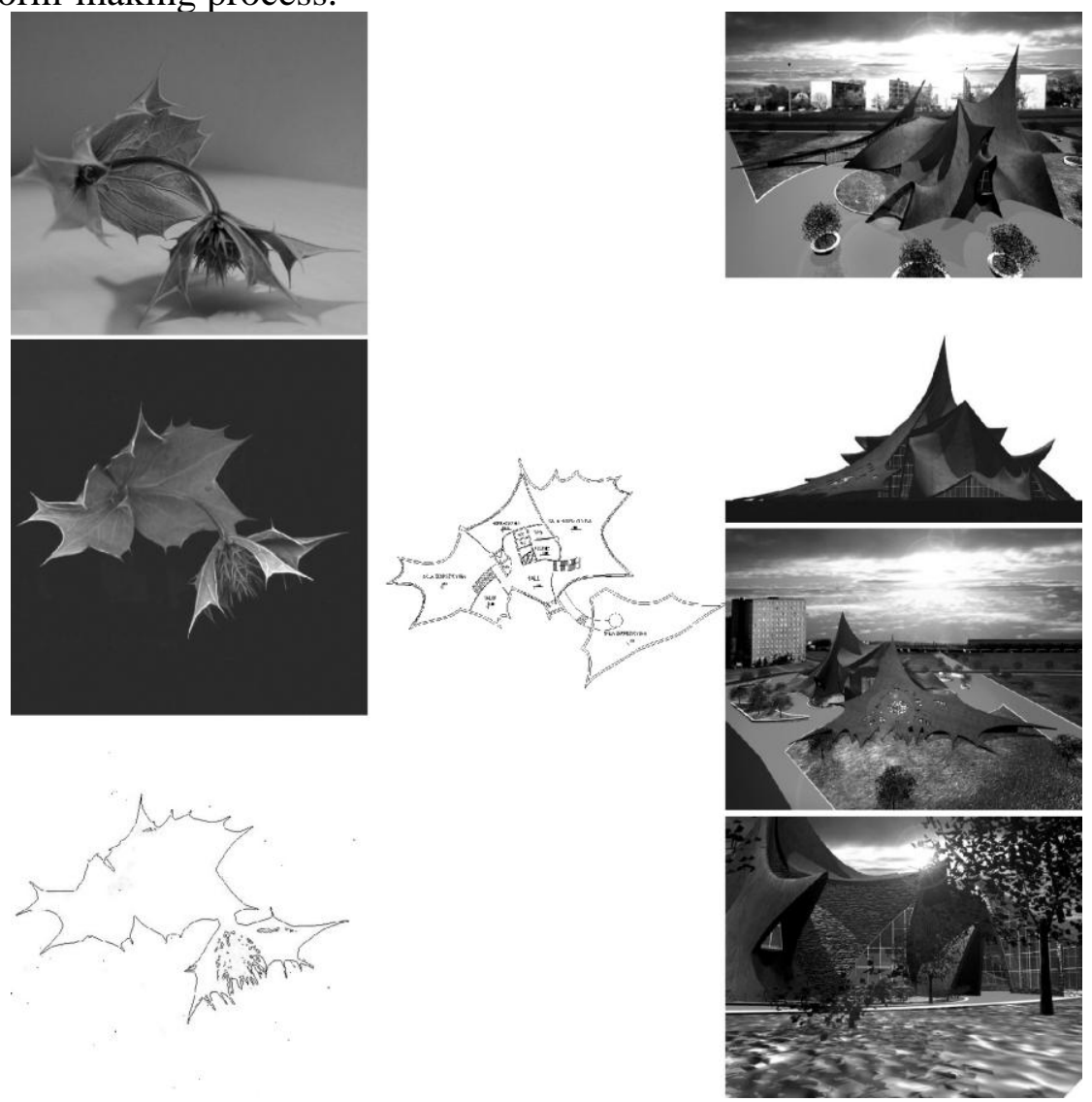

Fig. 3: Process of form-making using analogy of a sea-holly leaf

\subsubsection{Using metaphorization}

1 Halifax:" Organic Architecture and Beyond "Riverside Architectural Press; Tuns Press, 2008. Print. 
Many theories of creativity (theory of incubation elaborated by Wallas, genploration (Finke, Ward and Smith), redundant generation (Lem), synectics (Gordon)) emphasize the role of unpredictable associations and metaphors in creativity. Process of metaphorization is characteristic for our era and plays important role in creative process. As a confirmation of this a group of students were given Rodtchenko's pictures as an inspiration for a form-making process (Fig.4). On the basis of these pictures each student elaborated an abstract 2D composition and transformed it into a plan of a gallery. From one Rodtchenko's picture different students created totally different sketches, and in a consequence different unpredicted 3D forms. ${ }^{2}$

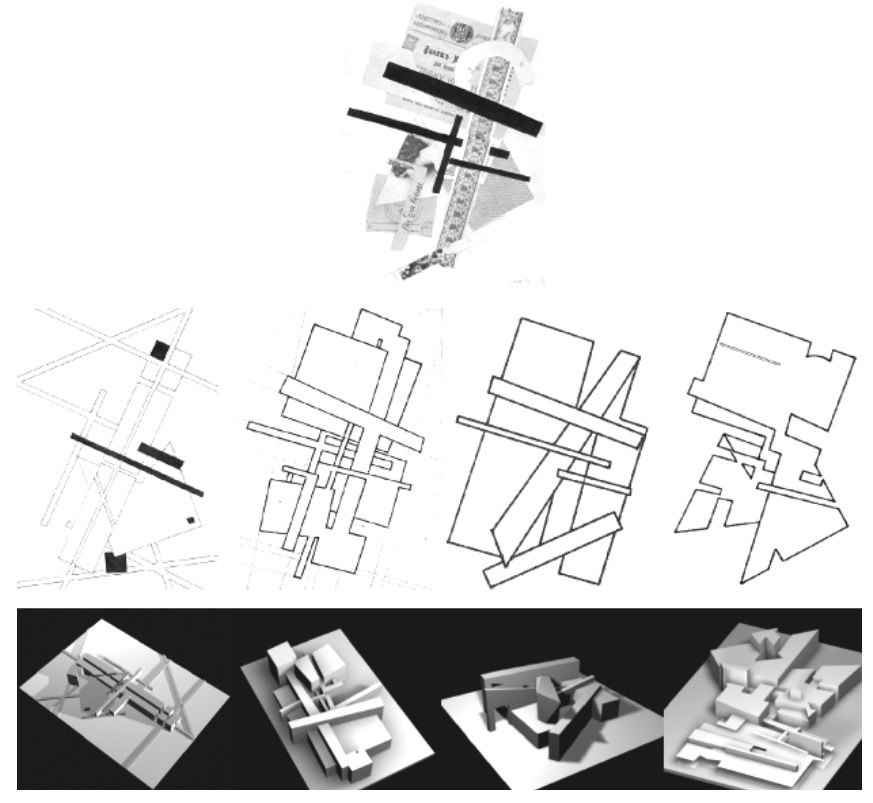

Fig. 4: Process of form-making using metaphorization of Rodtchenko's picture.

\subsection{Digital Form-making}

Digital systems have emerged as a fundamental keystone in architectural design during the last decades $^{3 .}$ The development of computer-aided design can be categorized into two main areas; "the representation and production of the geometry and topology of designed objects" and "the representation and use of knowledge to support or carry the synthesis of designs".

Examples of computational form seeking without logic:

\subsubsection{Using Fuzzy Modeling}

In the creation of the architectural form, the first drawing is a fuzzy, imprecise image of the object, barely reflecting the general outline of the idea. If we are doing conceptual work, it is more difficult to make a hint or suggestion of something with the computer than when doing it by hand. It is extremely difficult to create a drawing that hints at a basic form or idea.

With fuzzy modeling, the form of design is expressed in less crisp, essentially similar to the manners of architectural sketching with adding tolerance. Fuzzy logic Is a form of many-valued logic; it deals with reasoning that is approximate rather than fixed and exact. Compared to traditional binary sets (where variables may take on true or false values).

\subsubsection{Using Random Function}

2 Alexander Asanowicz: "Computer, Creativity and Unpredictability" in Digital Design Methods, 350-354.

3 DİNO, GÜRSEL İpek. "Creative design exploration by parametric generative systems in architecture", ODTÜ, 2012. 
The Webster dictionary defines randomness as "lacking a definite plan, purpose, or pattern. In this method of creation forms are not born in the architect's mind but they are produced by the computer. The architect only chooses, evaluates and transforms this form. ${ }^{4}$

Searching for the idea in the buzzing, i.e. where we have just thought that there is nothing there, has become a new creation process. The theory of chaos makes it possible to search for the form in the chaos of incidental elements or systems with the use of random function (Fig.5), which helps generate both two-and three-dimensional spatial structures. ${ }^{5}$

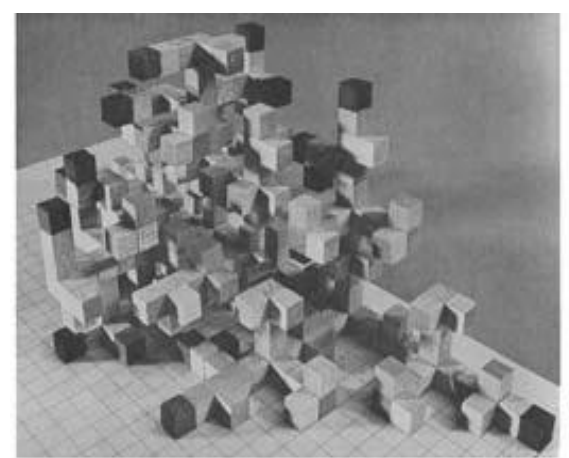

a.
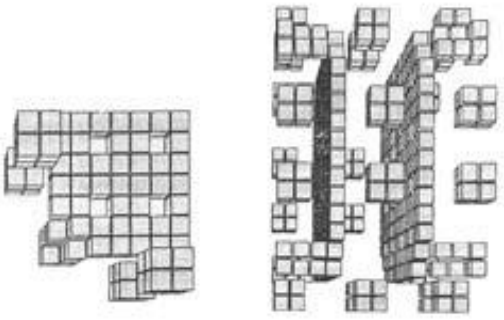

$b$.
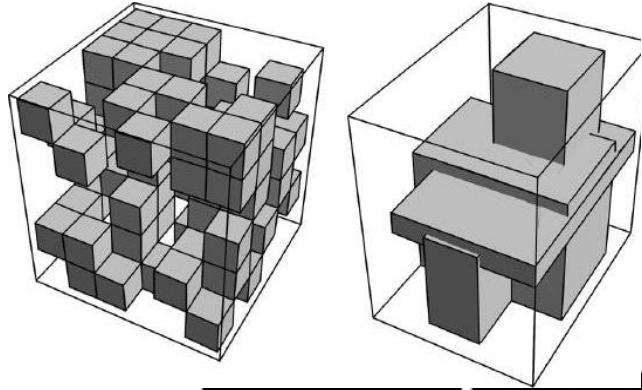
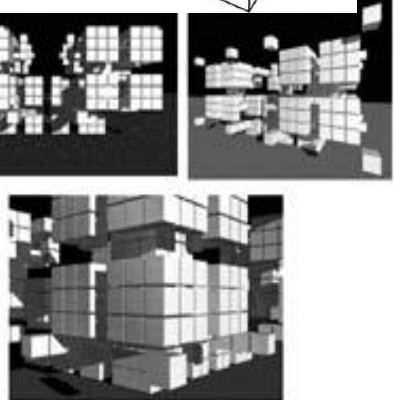

c.

Fig. 5: Form generated using iterated random function

\subsubsection{Using 3D Digitizing}

The availability of 3D digitizing or scanning affords a greater freedom of movement between real and digital modeling environments. The relationship between "real" and "virtual" domains is as intimate as the designer may wish. For example, Gehry uses 3D digitizers to transcribe the formal surface qualities of handmade models directly to the computer (Fig.6). He began modeling sculpture in wood and metal. With the CAD software Catia, he then developed models for the woven steel surface panels and supporting steel strakes of the sculpture. ${ }^{6}$
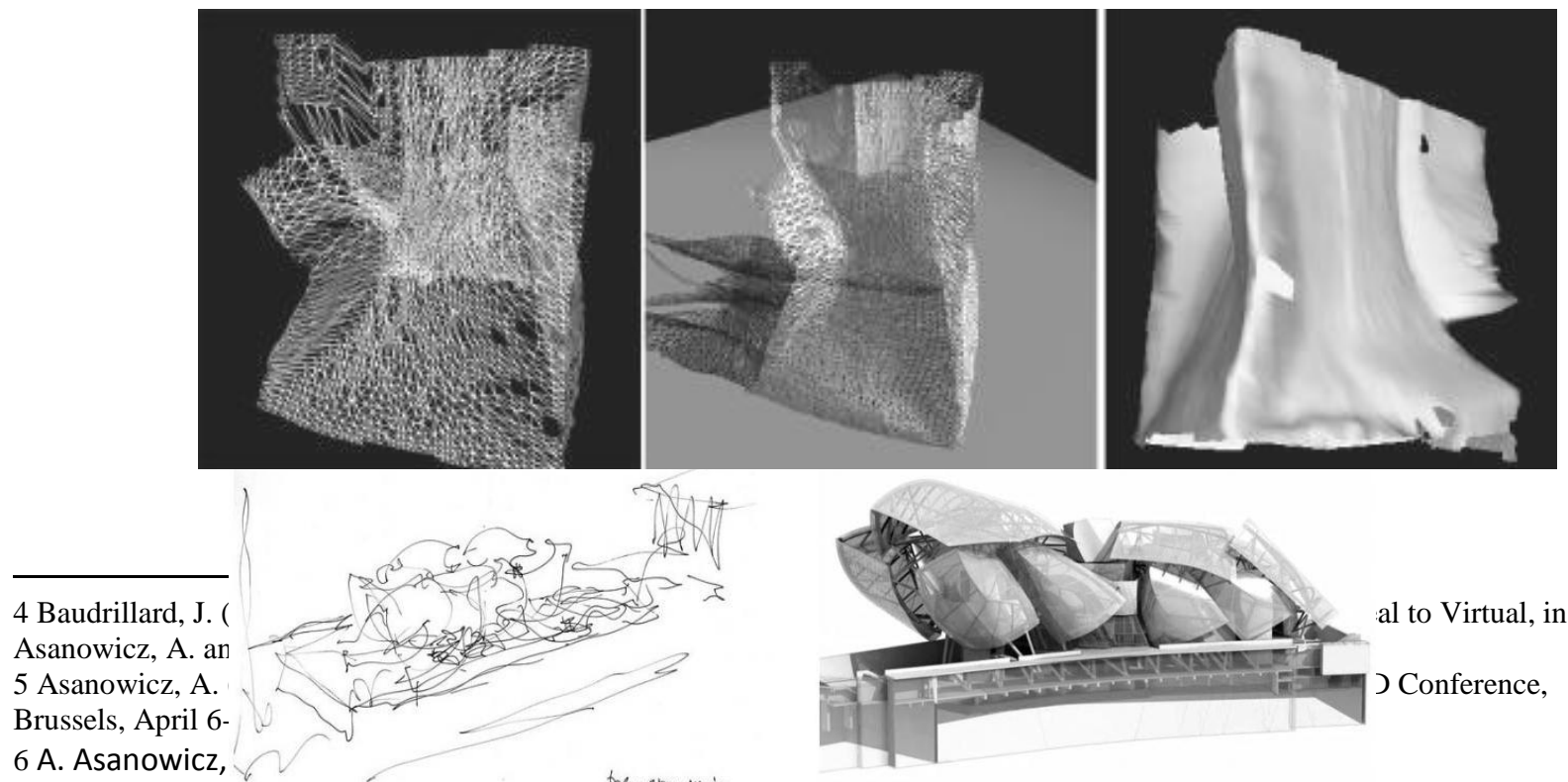

bran then migi
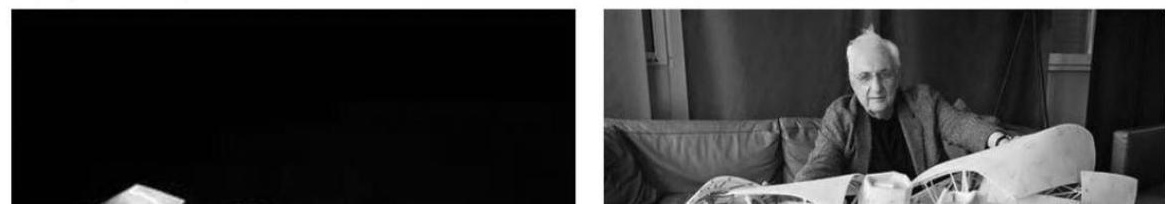
Fig.6: Gehry's Form-making process using 3D scanning transformation

\section{Form-Finding}

Form Finding is a process of discovery and editing; form exclusively determined by a function and resulted from applying rules, constraints, parametric dependencies, genetic structures, case-bases or defined algorithms within a medium (Fig.7). We will call this medium the "generator" that could be an analogue form finding generator or digital form finding generator. Also extreme form-finding is not architecture but applied engineering.

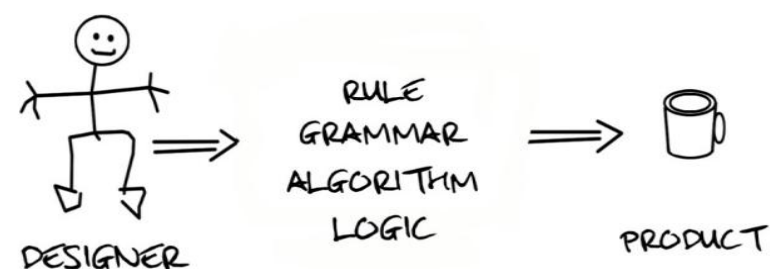

Fig.7: form finding process

\subsection{Analogue Form -finding}

\subsubsection{Using Mathematical Rules}

Early attempts to analogue form-finding is rooted to Jean-Nicolas-Louis Durand followed an analogue form finding approach for the creation of neo-classical architecture by applying different combinations of building elements (Fig.8). Louis Sullivan's plates that describe processes for reproducing floral ornamentation based on geometrical constructs (Fig.9).

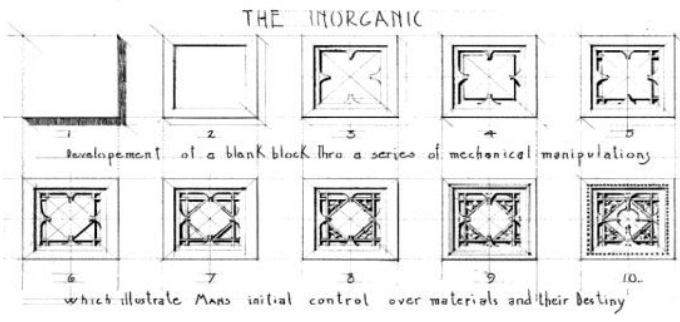

Fig.8: using mathematical rules in a form finding process 
THE INORGATIC:

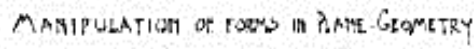

TEBLL GEOMETRY
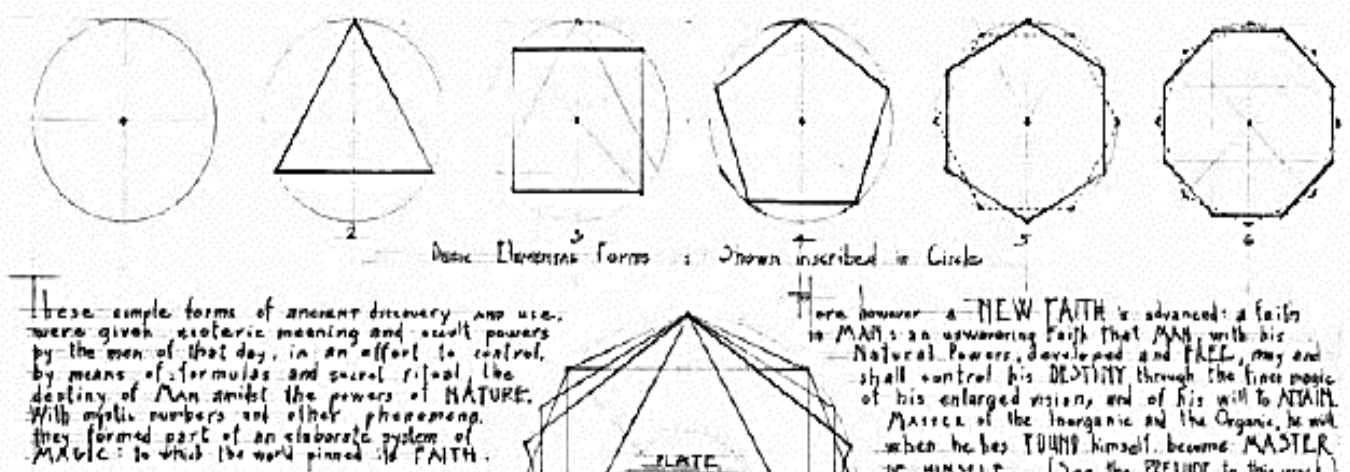

nustited in Ciste

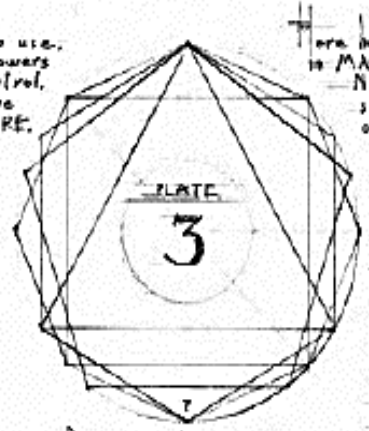

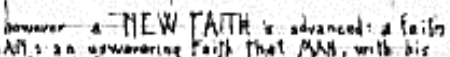

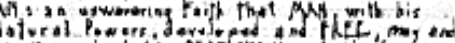

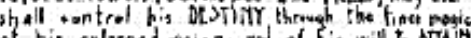

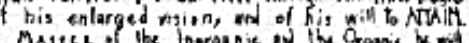
Marces of the therganic on the Organi. He wh

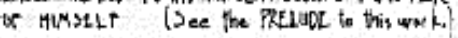

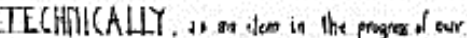
Demenstation, the thave forms, rigid in their quatity, are to be considered, in our polilosphy, 2 s

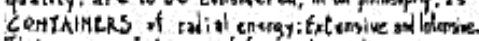

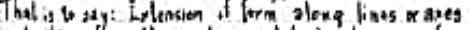
ualisting trem the conler and Lort iptenim of

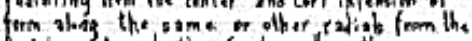

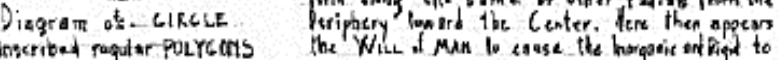
with inscribad paguiter POLYCers

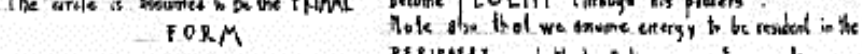
peribater. and that an lines are Linergy. lints. This nen be called PLASTIC GLOMETRY
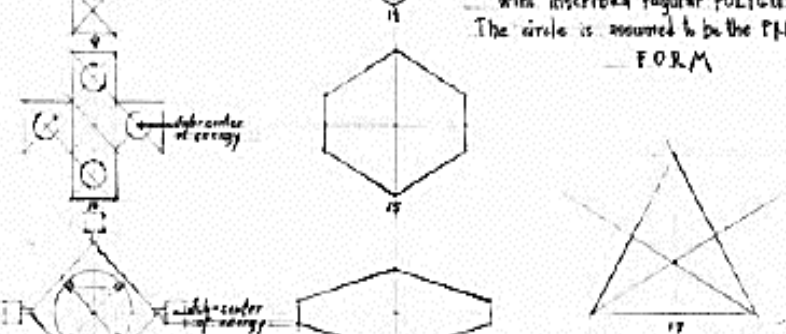

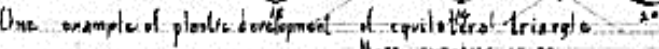
Hore svo-nas is 20
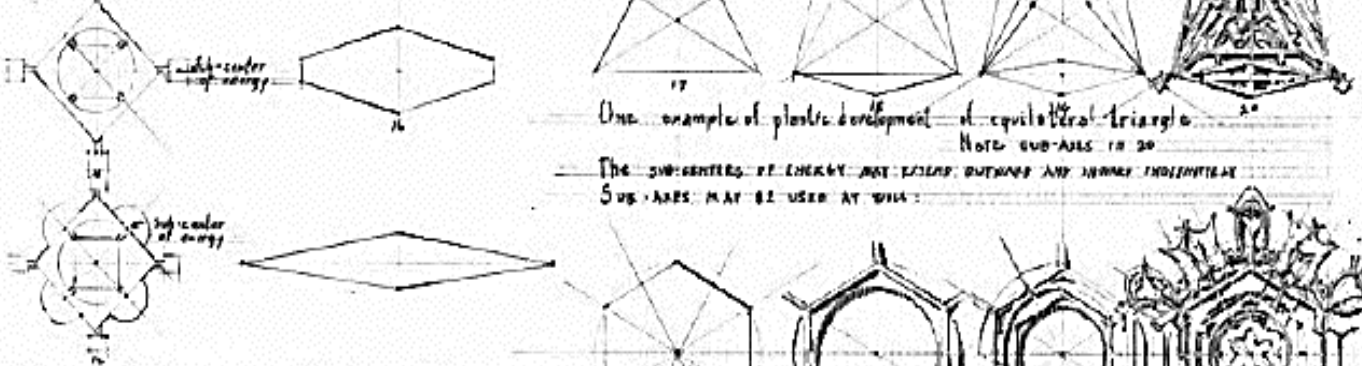

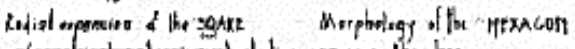

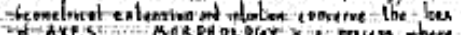

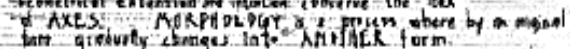

\& 8

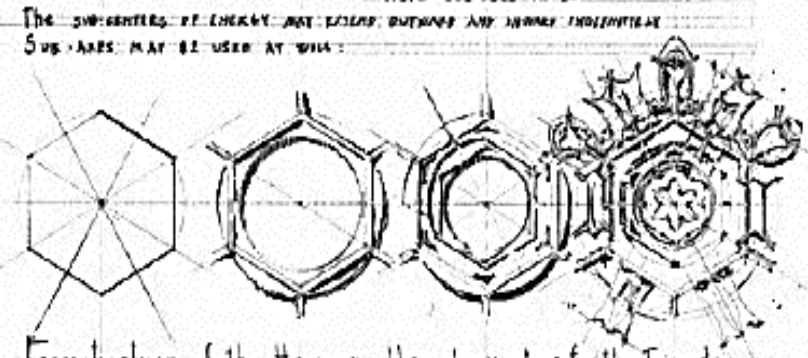

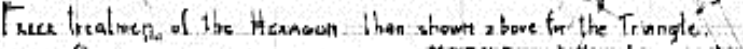

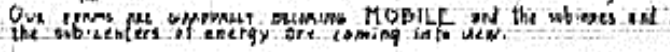

Fig.9: form finding process using fluent geometry

\subsubsection{Using Defined Style or Function}

Also Le Corbusier's Five Points of Architecture (Fig.10) in which he formalized his style are accounted as examples of analogue generative systems before the use of computation in architecture by El-Khalid.

7 Boesiger, W. : Le Corbusier, Ingoprint, Barcelona, 1992. 


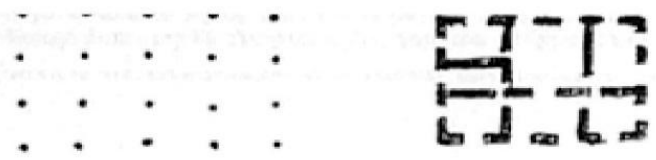

1. Columns, rather than walls.
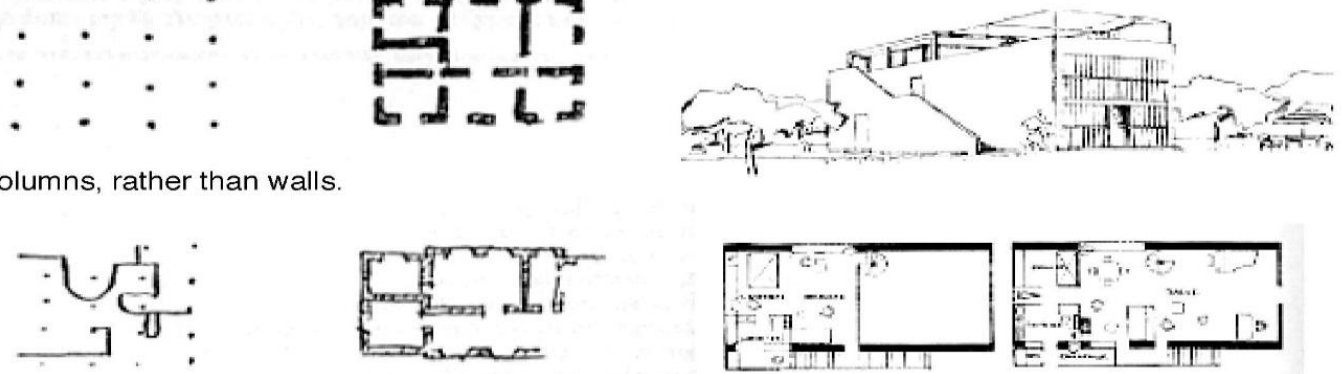

2. Free plan, rather than rooms.
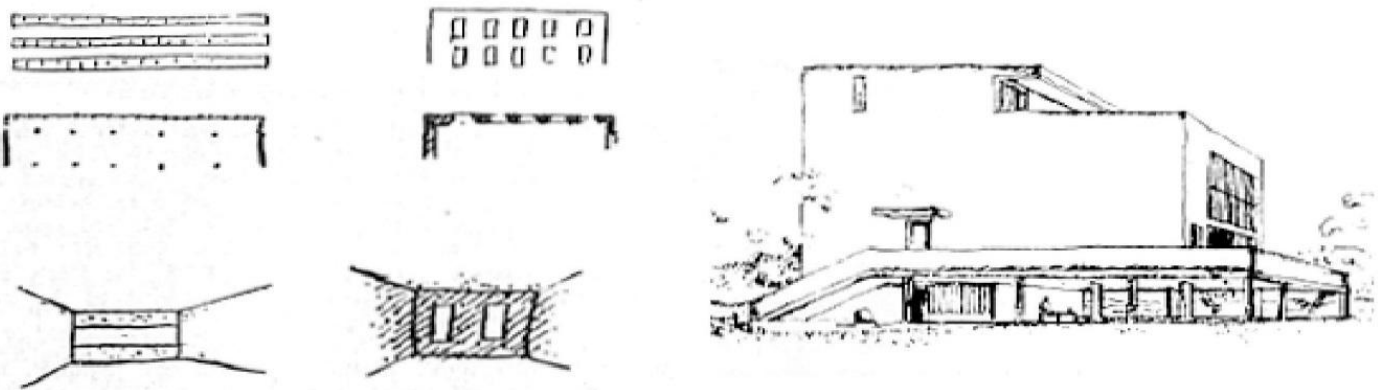

3. Window design freed from structural dependence: permits the long ribbon window.
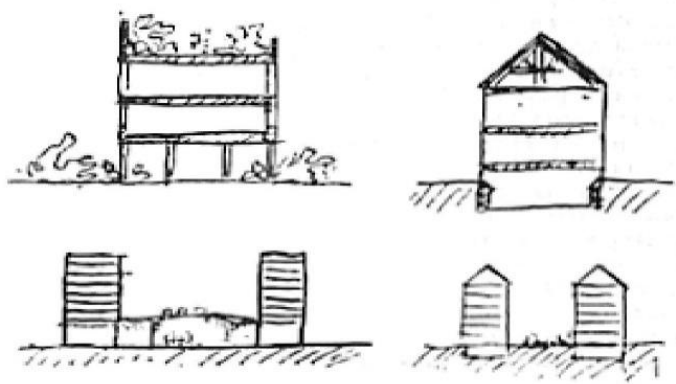

4. Pilotis elevate the building off the ground

5. Occupiable roof terrace, rather than pithched roof: restores the area of ground covered by the house.

Fig. 10: Le Corbusier's 5 points

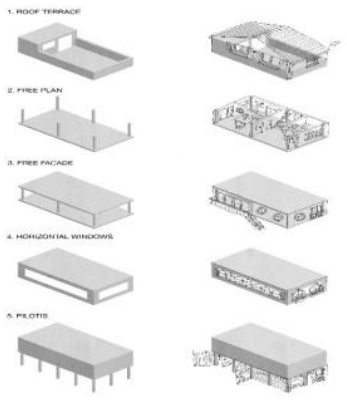

\subsubsection{Using Transformational Rules}

A recent example is Peter Eisenman, who used analogue transformational rules in architectural design synthesis. Eisenman's design concept operates on a system (a language) that permits creative action, generating an infinite number of utterances and making infinite use of finite means (Fig.11). Eisenman reflects this practice on the design of a series of houses (House II - X). ${ }^{8}$ 


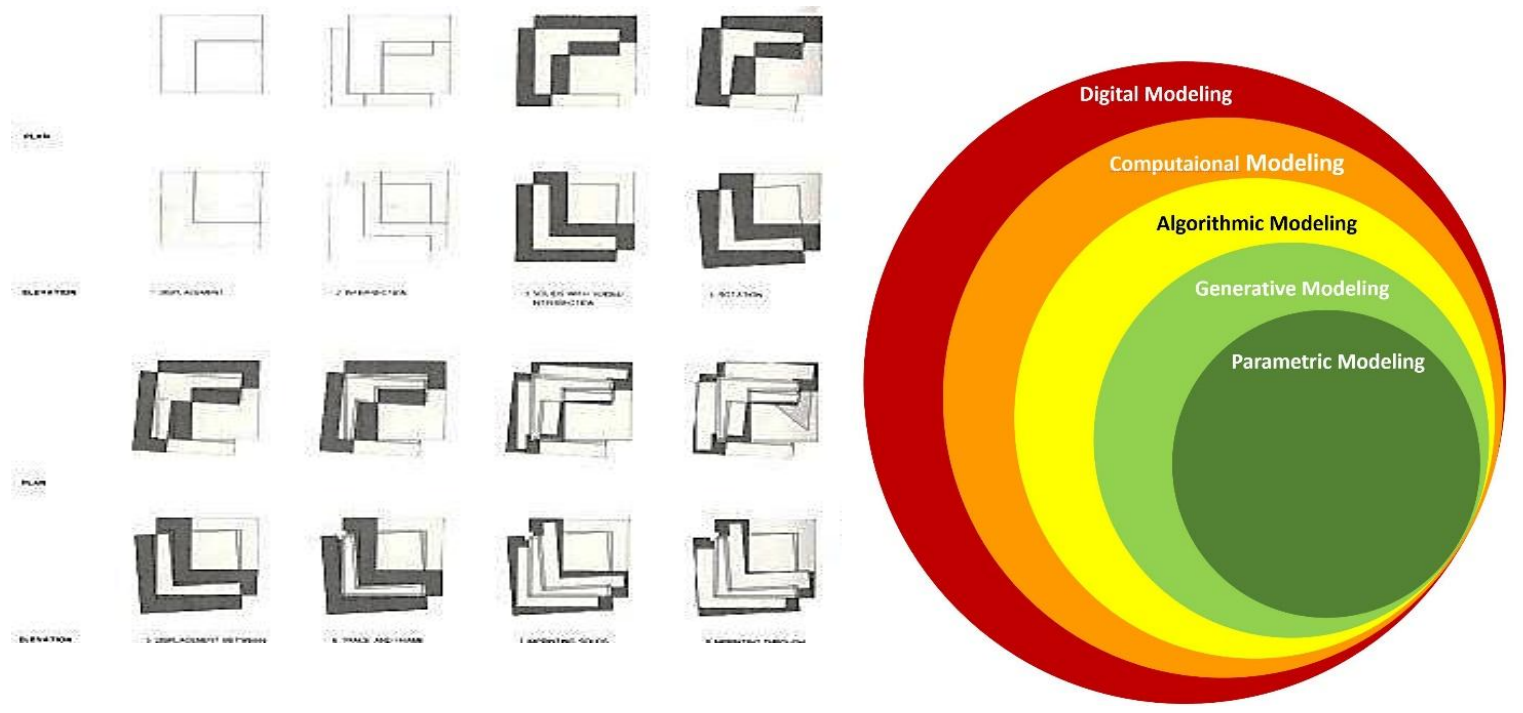

Fig.11: using transformational rules to form plans

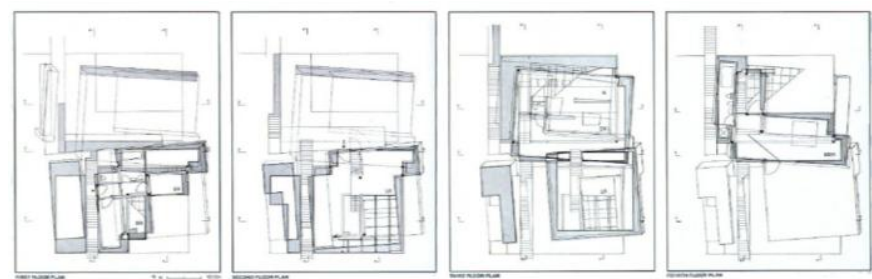

Fig.12: Digital Modeling hierarchy

\subsection{Digital Form-finding}

Digital form-finding can be used to generate orthogonal conventional forms using basic shape algebra and formal logic or complex three-dimensional curves and folding surfaces using trigonometric parameterized functions. Computational algorithmic modeling is a run-end process that apply rules and algorithms within a computational medium but without a potential of changing a rule with direct manipulation applied to the end result. If the process offers this feedback loop, it is a form generation process whether generative or parametric that will be next discussed (Fig.12).

\subsubsection{Computational Modeling}

Computational Modeling is the way to create shapes which are not directly defined but rather they are the result of some form of computation and simulation through using computers (Fig.13).
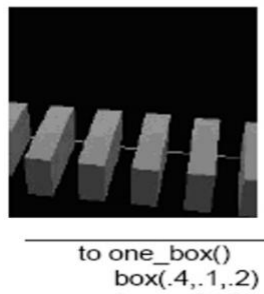

$$
\text { forward (.2) }
$$
end

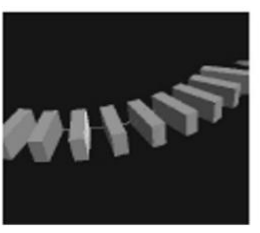

to one_box()

box $(.4,1, .2)$ forward (.2) end

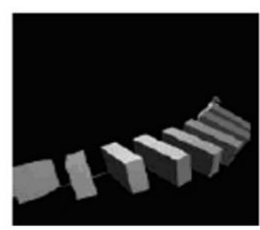

to one_box()

$\operatorname{box}(.4,1, .2)$

forward (.2) right(10) end

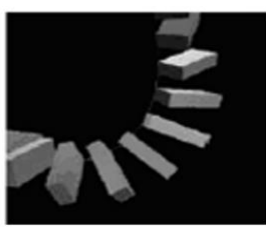

to one_box()

box $(.4, .1, .2)$

forward (.2)

right(20)

roll(20) pitch(20)

Fig.13: ways of using computational modeling

\subsubsection{Algorithmic Modeling}


An algorithm is a finite set of instructions that aim to fulfill a clearly defined purpose in a finite number of steps. ${ }^{9}$ An algorithm takes one value or a set of values as input, executes a series of computational steps that transform the input, and finally produces one value or a set of values as output (Fig.14). Processes are central and recorded but could not be tweaked. You will directly create algorithm yourself, rather than computer recording (and replaying) what you do with the mouse. Algorithms are not a special type of operation, necessarily. They are conceptual, a set of steps that you take in code to reach a specific goal. Algorithms have been commonly defined in simple terms as "instructions for completing a task" (Fig.15).

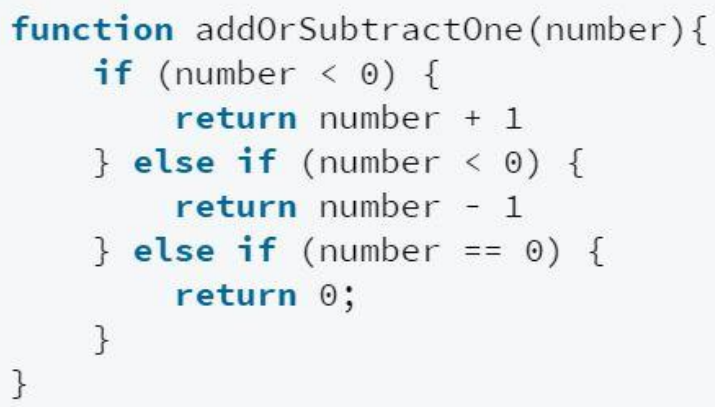

Fig.14: Algorithm example

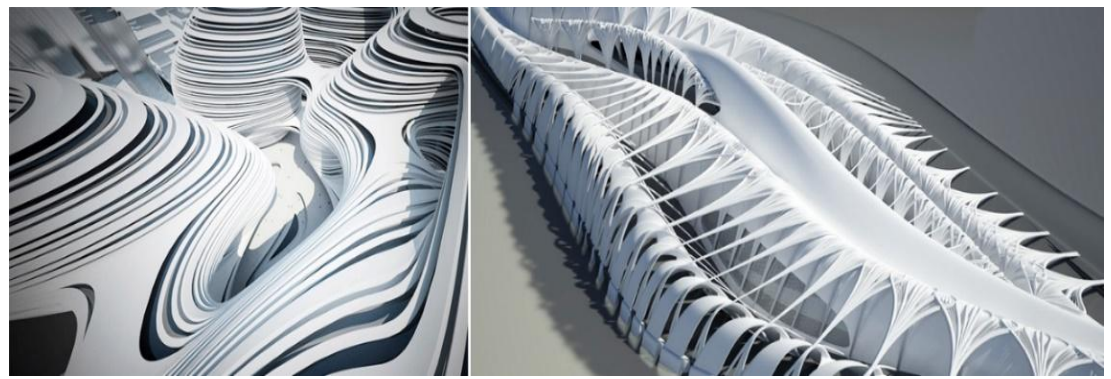

Fig.15: Algorithmic model

\subsubsection{Generative Modeling}

It is a design method in which the output is generated by a set of rules or an algorithm normally by using a computer program. Generative design is not about designing a building, its' about designing the system that builds a building (Fig.16).

Two important and distinguishing points that make a computational model generative; the rule or logic and the feedback loop, where the model takes its own output for input, to relatively generate complex surprising and unpredictable results. The solution is developed during several iterations of design operations. 


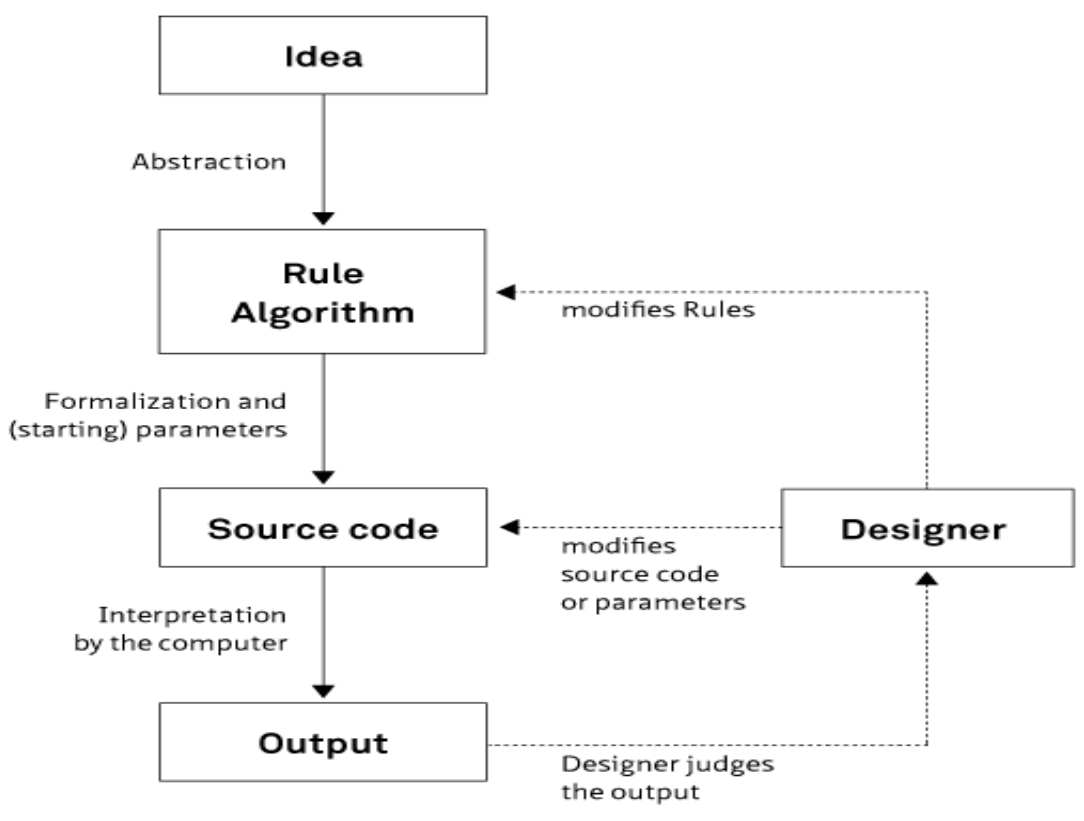

Fig.16 generative modeling process

A generative system is a production system that does not specify the design artifact, but instead specifies a higher-level specification that encodes the "making" of the artifact, or the design procedure. Therefore, generative systems are said to precede formation over form, which indicates a fundamental shift from the modeling of a designed "object" to modeling of the design's "logic". However, this does not mean that the generative system now becomes the designer, but that the human designer externalizes and encodes some of its working intelligence into the "generator" to carry out certain design tasks or solve problems.

Several generative design systems have been developed, well known tools such as shape grammars, parametric variations and algorithmic generation, and recent tools for searching of form in the world of chaos or random functions like Fractals and L-systems, and evolutionary tools based on the genetic engineering process like Genetic Algorithms and Cellular Automata. Generative design systems can be classified into three categories: linguistic, biological and parametric.

\section{$\circ \quad$ Linguistic Generative System}

A linguistic system is a grammar-based formalism where a set of compositional rules (syntax) govern and shape the design (semantics). The computational implementation of linguistic generative systems primarily manifests itself in shape grammars, L-systems and Fractals. A Brief will be given for each:

\section{- Shape Grammars}

Shape grammars were first introduced by Gips and Stiny in 1972, as an idea to describe visual shape compositions ${ }^{10}$. A shape grammar is a set of rules to specify how one shape or part of a shape can be replaced by another (Fig.17). This simple substitution process can be used to describe a certain design style or generate new ones ${ }^{11}$ (Fig.18).

10 Stiny, G.: Computing with Form and Meaning in Architecture. Journal of Architectural Education, 39(1): 7-19, 1985. 11 Knight, T.W.: Shape Grammars in Education and Practice: History and Prospects. Online paper, Department of Architecture, MIT, 2000. 

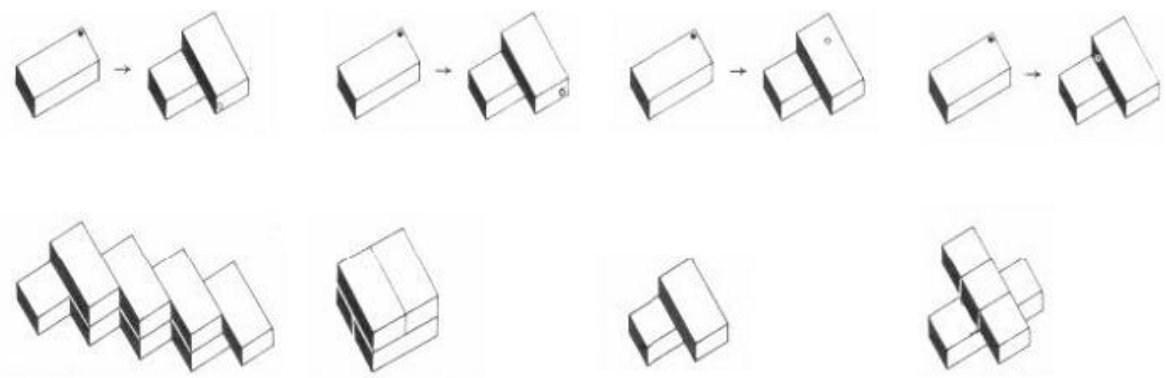

Fig. 17: Various 3D labeled rules for standard shape grammar and their derivations.
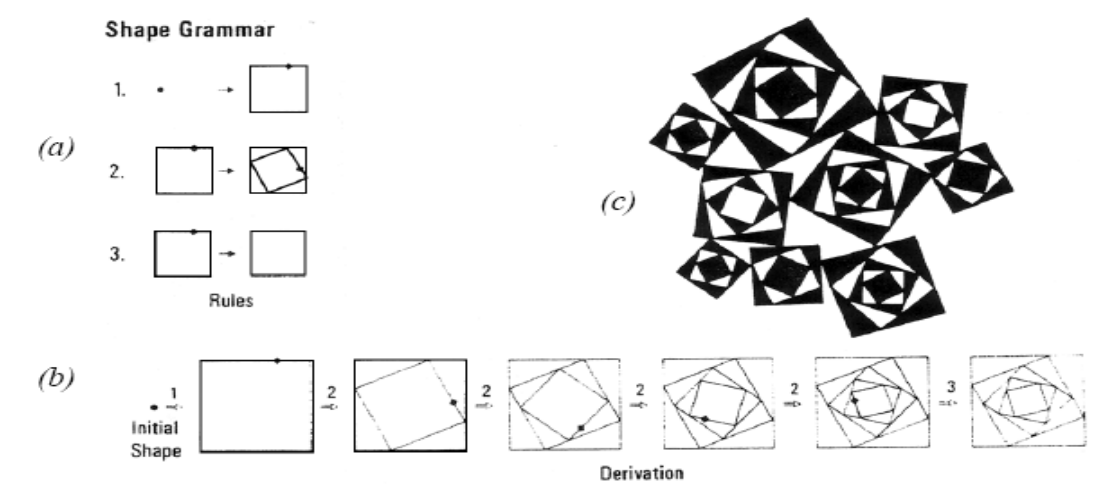

Fig.18: (a) Rules for a Standard Shape Grammar (b) A derivation of the rules (c) A result generated by applying the rules repeatedly

\section{- L-Systems}

L-systems were introduced by A. Lindenmayer as a mathematical formalism for modeling multicellular organisms that form linear or branching filaments (Fig.19). The whole organism is treated as an assembly of discrete units, called modules. Each module is represented by a symbol (a letter of the L-system alphabet), which specifies the module's type. In addition, a module can be characterized by one or more numerically-valued parameters, which, together with the symbol, characterize the module's state. The resulting concept of parametric L-systems has been formalized in, among others.

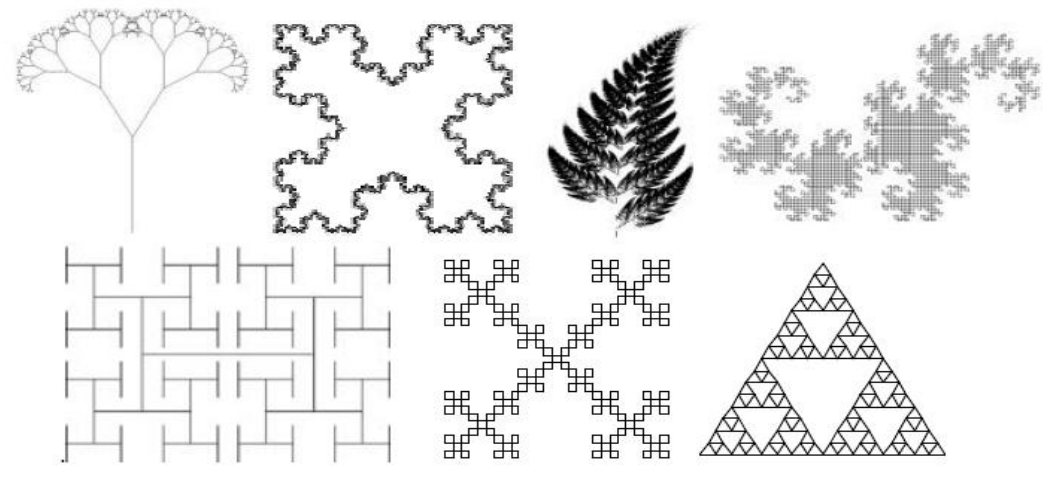

Fig.19: Example image from L-Systems in Architecture

12 Shakiban C. and Berstedt J.E.: Generalized Koch Snowflakes. In Bridges: Mathematical Connections in Art, Music and Science, 1998. 


\section{- Fractals}

The term fractal comes from the Latin word fractus, which means broken and irregular. Its own name indicates that it is a geometry that is specially thought for irregular objects -a geometry of nature- in contrast with the regular geometry created by men. A fractal is an object or quantity that displays self-similarity on all scales with non-integer dimensions (Fig. 20). The object need not exhibit exactly the same structure at all scales, but the same "type" of structures must appear on all scales. ${ }^{13}$
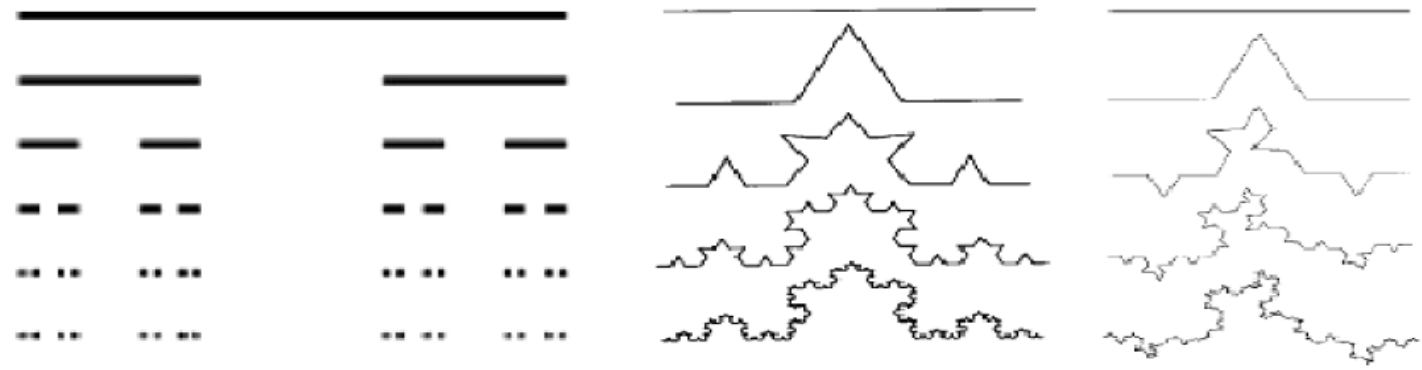

Fig. 20: Cantor set (left) and Koch curve (right)

The mathematical nature of fractals based on chaotic mathematical equations that have repeated manner (Fig.21). Chaotic processes are not random; they follow rules, but even very simple rules can produce extreme complexity (Fig.22). 14

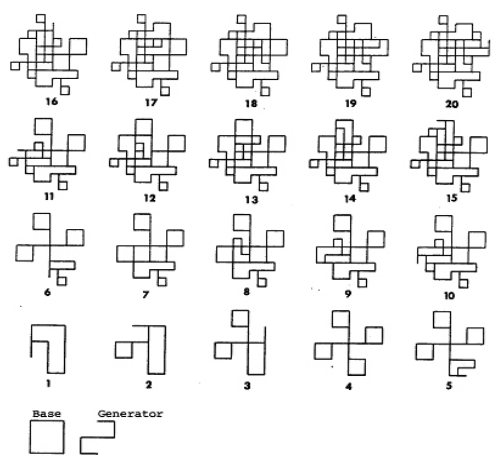

Fig. 21: A fractal process after 20 iteration

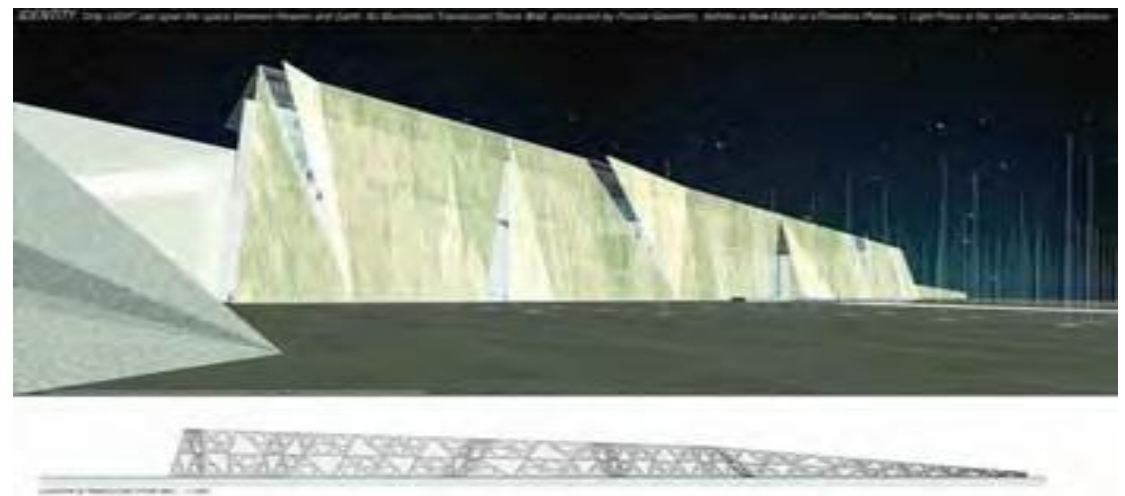

Fig. 22: Sierpinski set used in the translucent alabaster cladding of the grand Egyptian museum's façade

13 Bovill, C.: Fractal Geometry in Architecture and Design. Birkhauser, Boston, 1996.

14 Yessios, Chris I.: A Fractal Studio. In ACADIA ' 87 Workshop proceedings, 1987. 


\section{○ Biological System}

Biological generative design systems adopt a different generative strategy, which takes nature and complex living organisms as a precedent and applies its principles in the derivation and transformation of architectural form.

Since Charles Darwin proposed his theory stating that all species are generated via the process of evolution, Attempts had been developed to explain the adaptive process of natural systems and to design artificial systems based upon these natural ones (Fig.23).

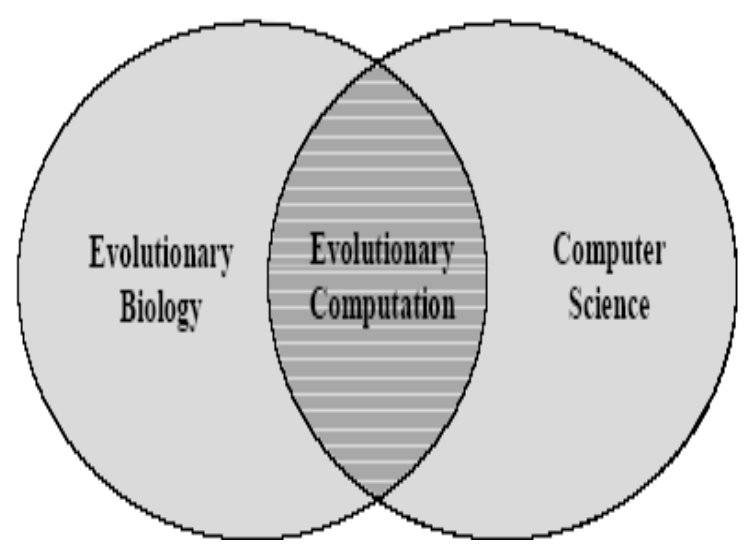

Fig. 23: Evolutionary computation roots in computer science

and Evolutionary Biology

\section{- Genetic Algorithm}

Genetic algorithms (GAs) are algorithms inspired from Darwin's theory of evolution. These algorithms operate with the population of candidate solutions (individuals). Every new population is formed using genetically inspired operators (like crossover and mutation) (Fig.24) and through a selection pressure, which guides the evolution towards better areas of the search space. The evolutionary algorithms receive this guidance by evaluating every candidate solution to define its fitness. The fitness, calculated by the fitness function (i.e. objective function), indicates how well the solution fulfills the problem objectives (Fig.25).

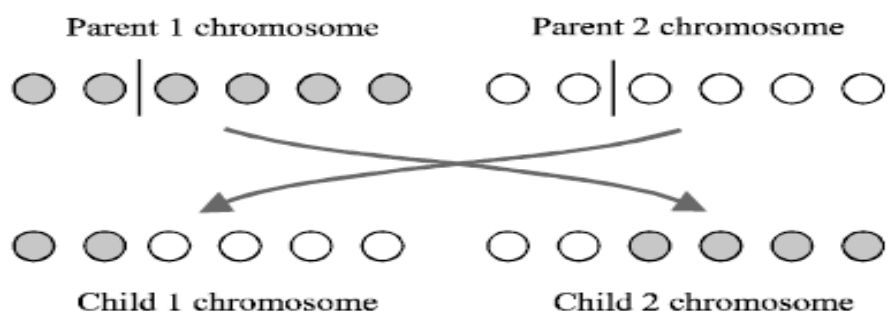

Fig.24: The behavior of crossover operator

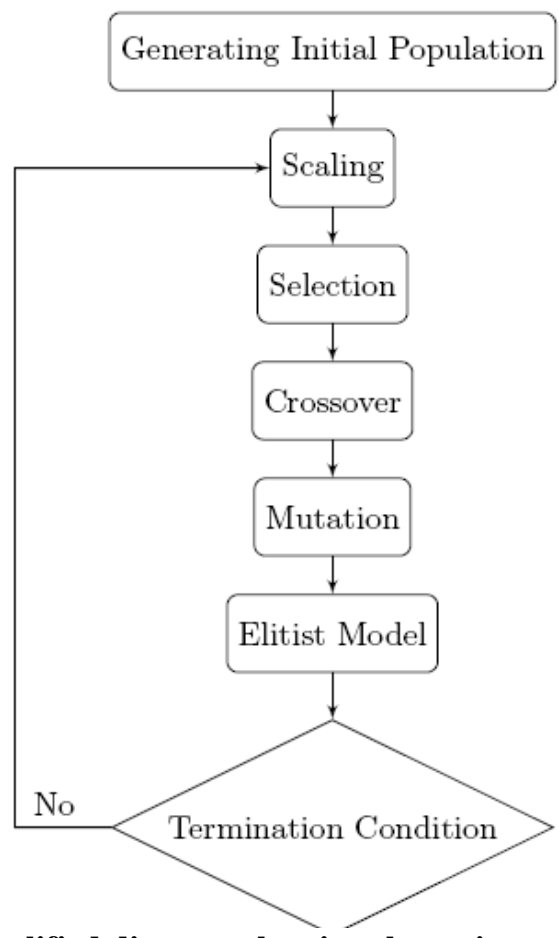

Fig.25: Simplified diagram showing the main steps of a genetic algorithm 
In GA each individual element (in the phenotype) in the solution space, takes a code (a binary code depends on its properties) in the search space (in the genotypes). Phenotypes usually consist of collections of parameters; Genotypes consist of coded versions of these parameters. A coded parameter is normally referred as a gene; with the values a gene can be known as alleles. A collection of genes in one genotype is often held internally as a string, and is known as a chromosome. (Fig.26) shows GA's concept. Soddu used artificial DNA's to roduce various art work recorded (Fig. 27). ${ }^{16}$

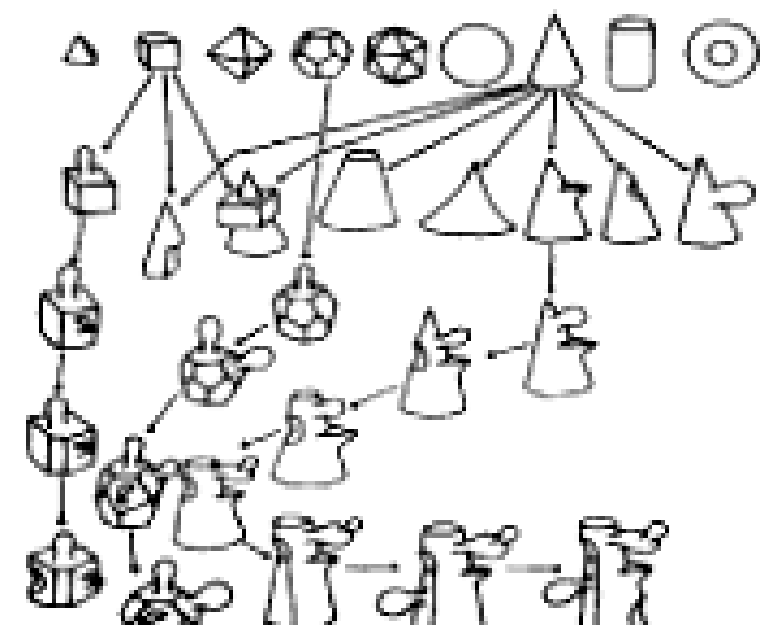

Fig. 26: Small hand-drawn genetic tree

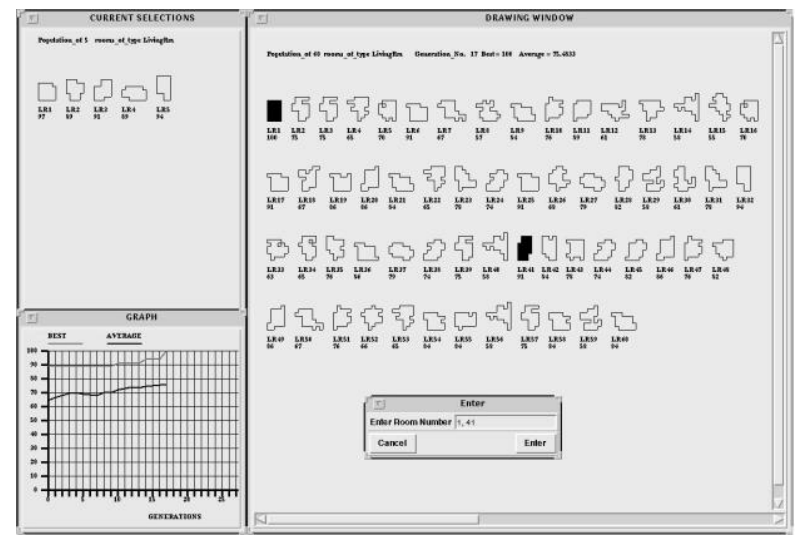

Fig. 27: Generated portraits “d'après Picasso" with “artificial DNA” by Soddu

\section{- Cellular Automata}

A cellular automaton is a collection of cells on a grid of specified shape that evolves through a number of discrete time steps according to a set of rules based on the states of neighboring cells (Fig.28).

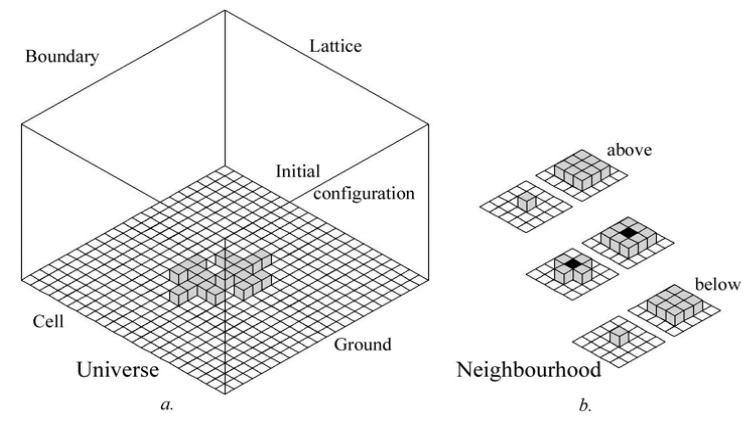

Fig. 28: Basic cellular automata terminology

16 Frazer John\& Frazer, Julia\& Xiyu, Liu\& Mingxi\& others: Generative and Evolutionary Techniques for Building Envelope Design. GA (Generative Art and Design Conference), 2002. 
The rules are then applied iteratively for as many time steps as desired. von Neumann was one of the first people to consider such a model, and incorporated a cellular model into his "universal constructor." Cellular automata were studied in the early 1950s as a possible model for biological systems. More comprehensive studies of cellular automata have been performed to produce complex shapes (Fig.29). ${ }^{17}$
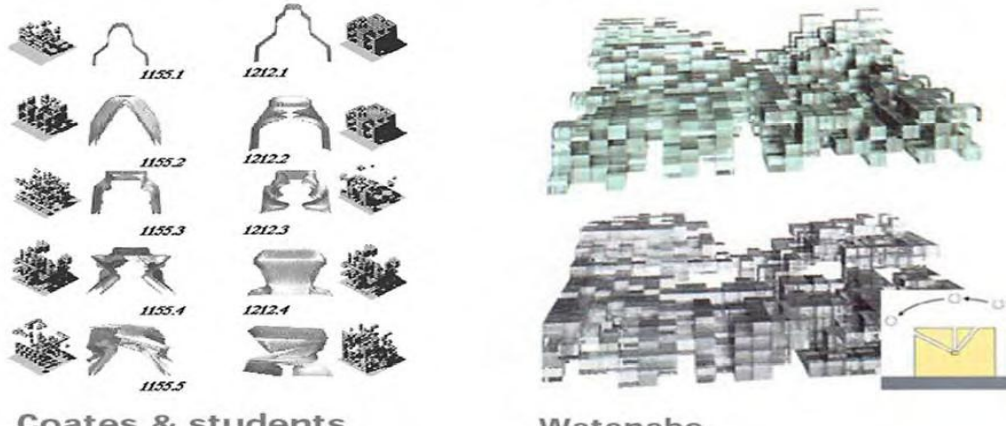

Vatanabe

\subsubsection{Parametric Modeling}

Fig. 29: Some interpretations of cellular automata

Parametric Modeling is turning design into a set of principles encoded as a sequence of parametric equations. The equations are used to express certain quantities as explicit functions of a number of variables. By changing any parameter in the equation new forms and new shapes could be generated (Fig.30).

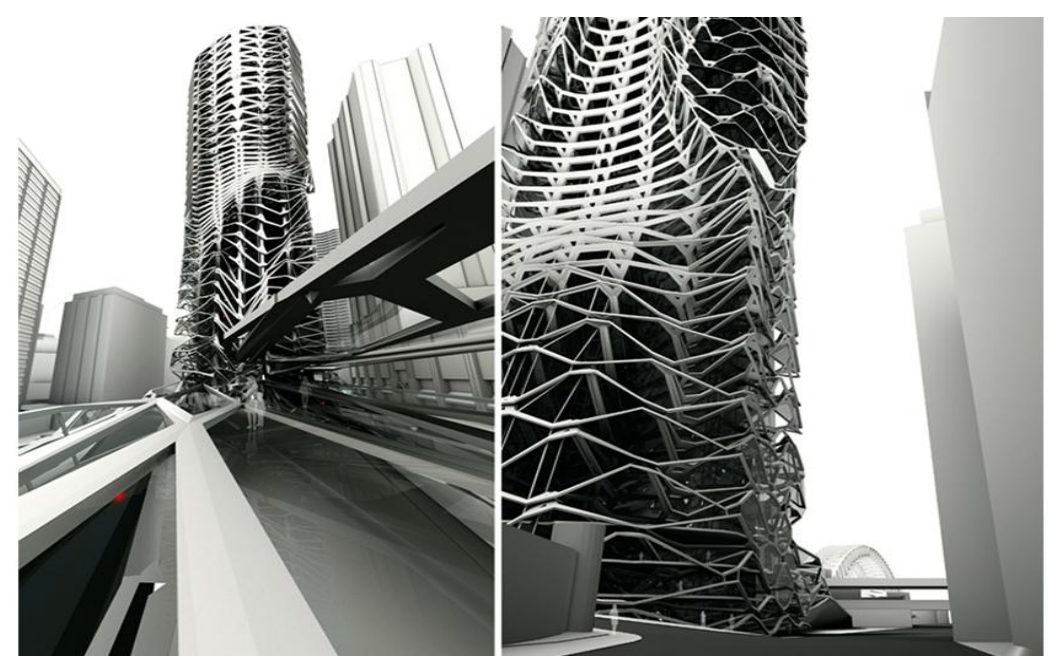

Fig. 30: Interpretation of parametric modeling.

Parametric design is a subcategory of algorithmic design, and is strictly based on an algorithmic construct. Computationally speaking, there is no difference between algorithmic and parametric systems; algorithms by default operate on parameters, and a parametric system's fundamental component is the algorithm itself, called the schema or definition. However, different than algorithmic design, parametric systems emphasize the explicit and direct manipulation of the parameter values in order to induce a change on the design artifact. This simple difference between a purely algorithmic versus parametric design manifests itself only during the design process, where the parameter values are changed by the designer in order to manipulate the

17 Krawczyk, Robert J.: Architectural Interpretation of Cellular Automata. Illinois Institute of Technology, College of Architecture, USA, Generative Art, 2002. 
design geometry in search of the optimal design solution. Parametric modeling can be classified to three generations, the first was a pure application for the parametric equations. This phase is the primary application for the concept of parametric design. It is based on mathematical equations that generate forms based on changing the equations or the variables (Fig. 31). By changing one of the variables or the equations, new forms will be generated. It reflects also the progression of the historical mathematical rules that constraints the proportions of the elevations, since the designer here generates the form based on his mathematical equations.

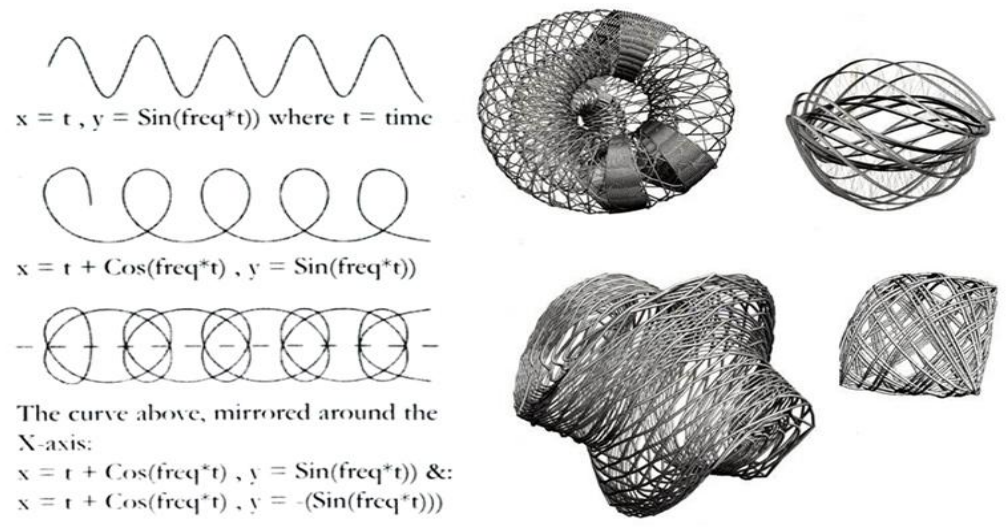

Fig. 31: forms generated by Mathematical equations in the first generation

The second generation is based on creating relationships between geometrical objects of a certain project, and by changing any dimension of one of the objects the whole design will be modified (Fig. 32). Parametric design in this generation suggests the use of parameters to generate a form but the greater importance here, are the underlying relations between elements of the form. Set of relationships between objects are maintained while the elements can be independently modified. 18
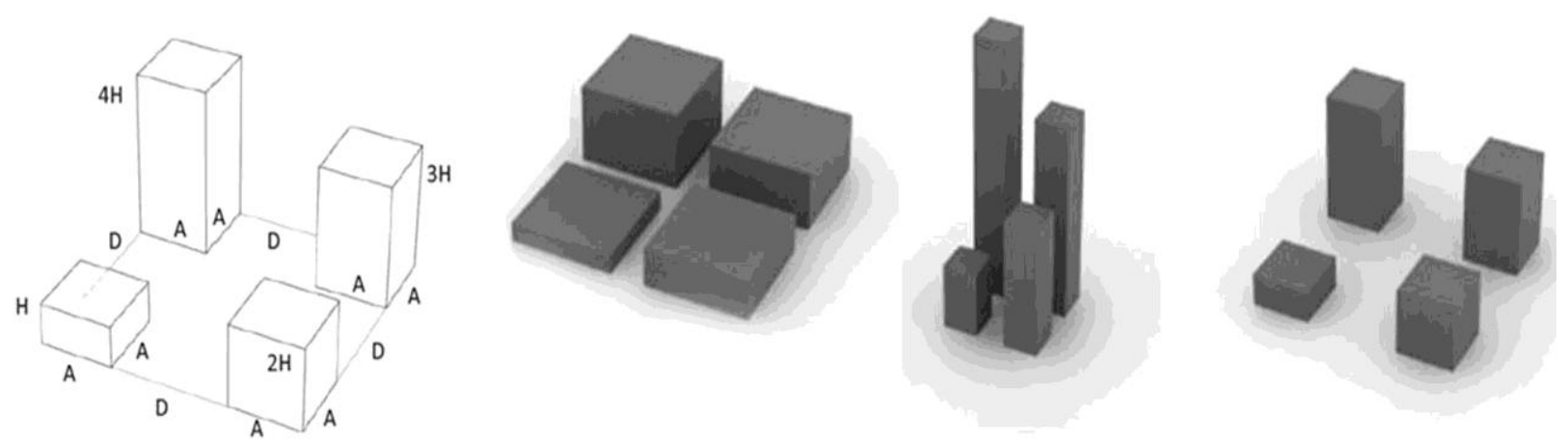

Fig. 32: The geometry of the four boxes is parametrically related to each other in the second generation

While the third generation in contemporary parametric design is based on designing an algorithm to generate the project. The parameters here will be the inputs of this algorithm, and by modifying the parameters new designs will be generated. It is similar to the previous phases but the different here is that the parameters are inputs in algorithms not in pure algebraic equations, which represents a wider spectrum for the idea of parametric design. The designer here starts to create his algorithm by determining certain procedures that at the end generates the final form which fulfills the designer rules (Fig. 33). 


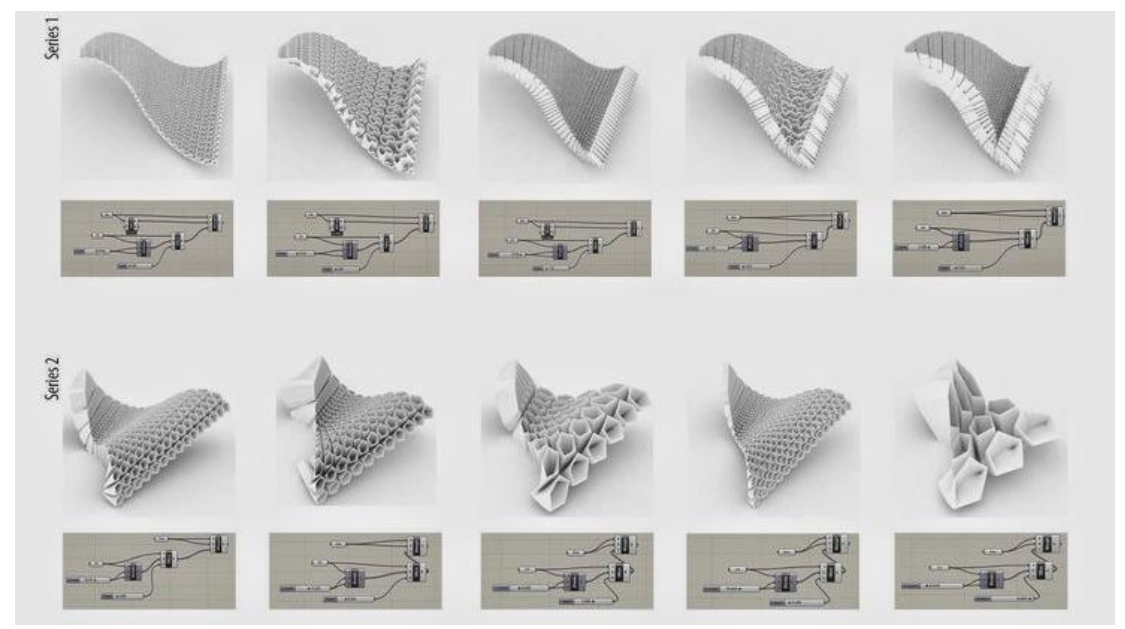

Fig. 33: various parametric surfaces generated using the third generation

The algorithmic steps could be used to create relations between geometrical entities similar to the previous parametric relations, or it may be based on mathematical equations. And by changing any of the input parameters new design variables will be generated. This generation represents clearly matured applications for using computers in the architectural designs. It reflects the full computational power of computers with their software and hardware (Fig. 34), It is different from the previous generations where most of the designs are not-realistic. Most of the designed projects of the first two generations, show showy forms reflecting information technology potentials without any concerns about solving real design problems. Recently, architects started to use common algorithmic steps such as Voronoi, A-star, Stochastic search, Tabu Search, Lsystems, Cellular Automata, Swarm intelligence, and Genetic Algorithms in their designs which consequently start to take part in their parametric designs. These common algorithms could be the main or part of the algorithmic process done by the architect to create his designs. Usually many architects create their parametric designs based mainly on one of the previously common algorithms.
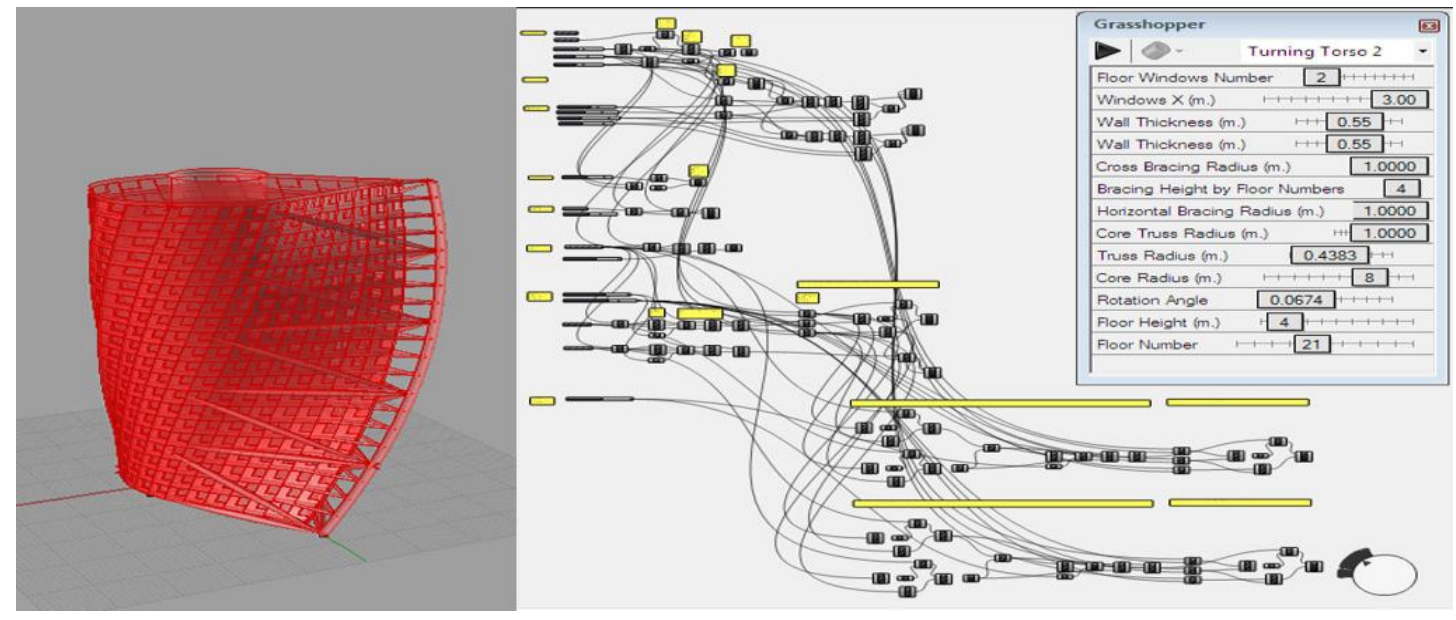


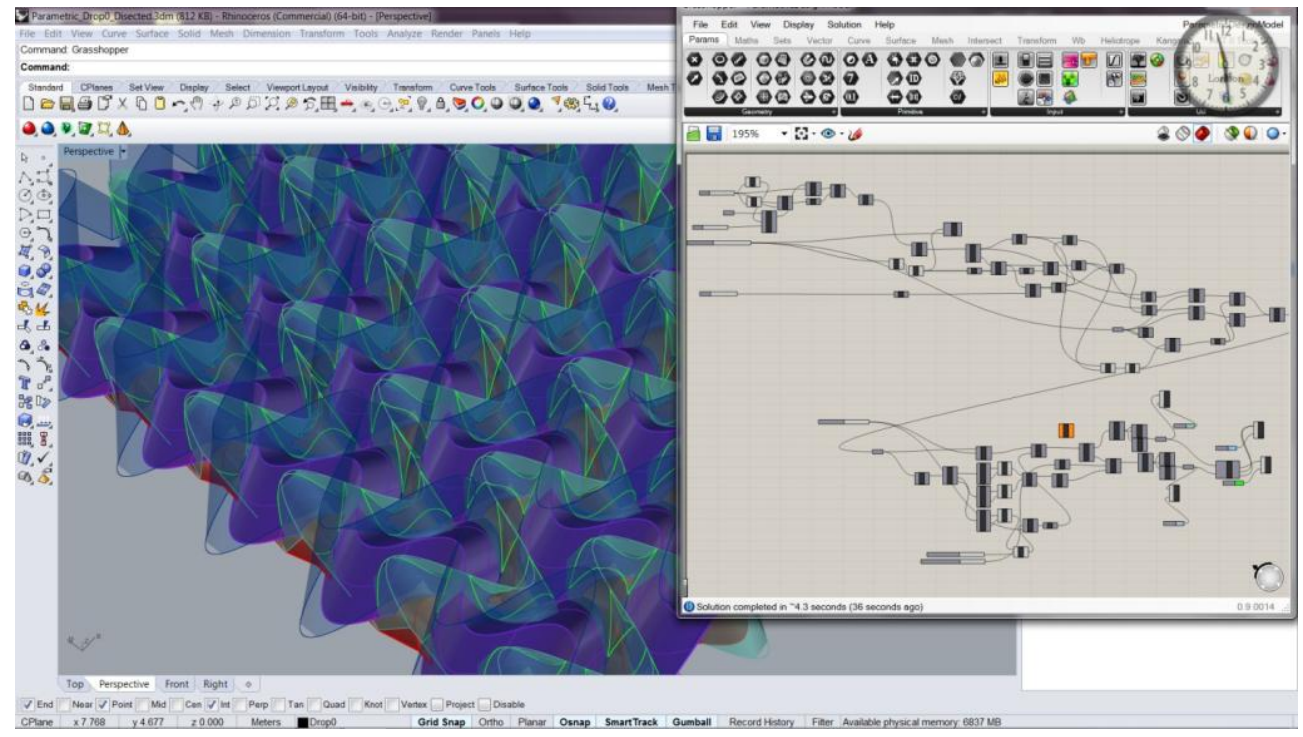

\section{CONCLUSION}

Fig. 34: Grasshopper interface with models manipulated

The following table discloses the main differences between form making and form finding:

\begin{tabular}{|c|c|c|}
\hline & Form-making & Form-finding \\
\hline Definition & $\begin{array}{l}\text { Form Making is a process of creation } \\
\text { using intuition, that is the ability to } \\
\text { foresee without trying to understand, } \\
\text { and imagination, that is the ability to } \\
\text { create certain images in our mind. } \\
\text { Form precedes analysis and design } \\
\text { constrains. }\end{array}$ & $\begin{array}{l}\text { Form Finding is a process of discovery and } \\
\text { editing; form exclusively determined by a } \\
\text { function and resulted from applying rules, } \\
\text { constraints, grammer, logic, genetic } \\
\text { structures, case-bases or defined algorithms } \\
\text { within a medium. }\end{array}$ \\
\hline $\begin{array}{l}\text { Methods of } \\
\text { applying }\end{array}$ & $\begin{array}{l}\text { Can be applied through: } \\
\text { 1. Analogue Form-making } \\
\text { - Using Coincidence. } \\
\text { - Using analogy and imitation of } \\
\text { nature. } \\
\text { - Using metaphorization. } \\
\text { 2. Digital Form-making } \\
\text { - Using Fuzzy Modeling. } \\
\text { - Using Random Function. } \\
\text { - Using 3D Digitizing. }\end{array}$ & $\begin{array}{l}\text { Can be applied through: } \\
\text { 1. Analogue Form-finding } \\
\text { - Using Mathematical Rules. } \\
\text { - Using Defined Style or Function } \\
\text { - Using Transformational Rules } \\
\text { 2. Digital Form-making } \\
\text { - Computational Modeling } \\
\text { - } \text { Algorithmic Modeling } \\
\text { - Generative Modeling } \\
\text { - Parametric Modeling }\end{array}$ \\
\hline
\end{tabular}




\begin{tabular}{|c|c|c|}
\hline $\begin{array}{l}\text { Applications } \\
\text { by architects }\end{array}$ & $\begin{array}{l}\text { 1. Analogue Form-making } \\
\text { Peter Stevens revealed the geometric } \\
\text { patterns present in nature. He } \\
\text { juxtaposes the branching of trees with } \\
\text { branching arteries and rivers; images } \\
\text { of crystal grains and soap bubbles. } \\
\text { 2. Digital Form-making } \\
\text { Gehry uses 3D digitizers to transcribe } \\
\text { the formal surface qualities of } \\
\text { handmade models directly to the } \\
\text { computer. He began modeling } \\
\text { sculpture in wood and metal. Then } \\
\text { with the CAD software Catia, he } \\
\text { develops models for his woven steel } \\
\text { surface panels. }\end{array}$ & $\begin{array}{l}\text { 1. Analogue Form-finding } \\
\text { Early attempts to analogue form-finding is } \\
\text { rooted to Jean-Nicolas-Louis Durand plates } \\
\text { who followed an analogue form finding } \\
\text { approach for the creation of neo-classical } \\
\text { architecture, obviously shown in his } \\
\text { ornamental plates. } \\
\text { 2. Digital Form-finding } \\
\text { Many architects and artist sail in this new } \\
\text { realm of digital form finding process, } \\
\text { whether using algorithmic, generative or } \\
\text { parametric methodologies like greg lynn and } \\
\text { cellestino soddu, shifting form from } \\
\text { following function, to the world of form } \\
\text { follows parameters. }\end{array}$ \\
\hline Disadv & $\begin{array}{l}\text { Extreme form-making is not } \\
\text { architecture but sculpture, perhaps } \\
\text { form without function. }\end{array}$ & $\begin{array}{l}\text { Extreme form-finding is not architecture but } \\
\text { applied engineering. }\end{array}$ \\
\hline
\end{tabular}

\section{REFERENCES}

1. Halifax:" Organic Architecture and Beyond "Riverside Architectural Press; Tuns Press, 2008. Print.

2. Alexander Asanowicz: "Computer, Creativity and Unpredictability" in Digital Design Methods, 350-354.

3. DİNO, GÜRSEL İpek. "Creative design exploration by parametric generative systems in architecture", ODTÜ, 2012.

4. Baudrillard, J. (1988) Simulacra and Simulations, in: Selected Writings, Sanford. Burry, M. (1996) From Real to Virtual, in Asanowicz, A. and A. Jakimowicz (eds.), Towards New Design Conventions, TUB, Bialystok, pp. 317-328.

5. Asanowicz, A. (2001) The End of Methodology - Towards New Integration, Proceedings of 3rd AVOCAAD Conference, Brussels, April 6-7, 2001, pp. 42-48

6. A. Asanowicz, Evolution of Media for Early Design Stages, Bialystok Poland.

7. Boesiger, W. : Le Corbusier, Ingoprint, Barcelona, 1992.

8. DINO, GÜRSEL İpek. "Creative design exploration by parametric generative systems in architecture", ODTÜ, 2012.

9. Gross, Mark D.: FormWriter A Little programming Language for Generating Three-Dimensional Form Algorithmically, CAAD futures.

10. Stiny, G.: Computing with Form and Meaning in Architecture. Journal of Architectural Education, 39(1): 719, 1985.

11. Knight, T.W.: Shape Grammars in Education and Practice: History and Prospects. Online paper, Department of Architecture, MIT, 2000.

12. Shakiban C. and Berstedt J.E.: Generalized Koch Snowflakes. In Bridges: Mathematical Connections in Art, Music and Science, 1998.

13. Bovill, C.: Fractal Geometry in Architecture and Design. Birkhauser, Boston, 1996.

14. Yessios, Chris I.: A Fractal Studio. In ACADIA ' 87 Workshop proceedings, 1987.

15. Bentley. Peter (Ed): Evolutionary design by computers. Morgan Kaufman publishers, 1999.

16. Frazer John\& Frazer, Julia\& Xiyu, Liu\& Mingxi\& others: Generative and Evolutionary Techniques for Building Envelope Design. GA (Generative Art and Design Conference), 2002.

17. Krawczyk, Robert J.: Architectural Interpretation of Cellular Automata. Illinois Institute of Technology, College of Architecture, USA, Generative Art, 2002.

18. Kotonik, Toni, "Algorithmic extension of architecture" master degree at ETHARCH/CAAD, Zurich,2006. 